\title{
Role of Magnetic Resonance Cholangiopancreatography in Malignant Obstructive Jaundice
}

\author{
Medhat M. Refat, Ahmed E Shaalan, Asmaa Sabry
}

Department of radiology, Benha faculty of medicine, Banha University, Egypt.

Correspondence to: Asmaa Sabry, Department of radiology, Benha faculty of medicine, Banha University, Egypt.

\section{Email:}

asmaskran0@gmail.com

Received: 14 February 2021

Accepted: 6 April 2021

\begin{abstract}
:
Background: Magnetic resonance cholangiopancreatography (MRCP) is a completely non-invasive technique that provides good projectional images without administration of contrast agents, without radiation, requires no anaethesia and less operator- dependent. AIM: The aim was to assess the role of magnetic resonance cholangiopancreatography (MRCP) in the diagnosis of malignant biliary obstruction. Patients and Methods: This study included 20 patients with malignant biliary obstruction diagnosed clinically, by ultrasonography and/or MDCT to do MRCP for better evaluation. Results: In our study, MRCP was accurate in the depiction the level of obstruction in the all 20 cases and showed $100 \%$ sensitivity, specificity and diagnostic accuracy in detecting the level of obstruction. MRCP showed significant preference in the ability to detect the cause of malignant obstruction; MRCP could diagnose the cause of malignant obstruction in $(90 \%)$ of cases. Within our study, MRCP
\end{abstract} detected other related malignant features in $40 \%$ of cases. Conclusion: Our data and in agreement with the previous studies has demonstrated that MRCP was accurate in the depiction the level of obstruction in the all 20 cases and showed 100\% sensitivity, specificity and diagnostic accuracy in detecting the level of obstruction with no substantial complications.

Keywords: MRCP, cholangiocarcinoma, obstructive jaundice, CT.

\section{Introduction:}

Jaundice is an indicator of significant underlying disease. It is caused by elevated serum bilirubin levels. It can be difficult to detect by physical examination alone.
Causes of non-obstructive jaundice include viral hepatitis, alcoholic liver disease, hemolysis and drug-induced liver injury. Obstructive jaundice may be due to benign 
causes as common bile duct stones or due to neoplastic causes ${ }^{[1]}$.

Malignant biliary obstruction (MBO) is a common disease observed in clinical practice. It is usually caused by cholangiocarcinoma, gall bladder and pancreatic malignancies, metastatic lymphadenopathy, hepatic and advanced gastric and duodenal malignancies ${ }^{[2]}$.

Cholangiocarcinoma and gallbladder carcinoma are the most common primary malignant tumors of the biliary system. Pancreatic cancer is a common cause for malignant biliary obstruction, often associated with dilatation of the pancreatic duct (double-duct sign). MRCP detect different pathologic entities, including cholangiocarcinoma, hepatocellular carcinoma, pancreatic carcinoma, distal common bile duct stricture, periampullary carcinoma, gallbladder carcinoma, lymphoma, metastasis, and suprarenal carcinoma. ${ }^{[3]}$.

The management of obstructive jaundice is often a difficult problem for the surgeon. Accurate information regarding the site, extent and nature of the obstructing lesion enables an early and precise decision on the type of surgical intervention necessary, and greatly improves the progress of these patients ${ }^{[4]}$.

The diagnosis of malignant biliary obstruction currently depends largely on radiographic imaging. Imaging provides necessary information for determining operability and the proper surgical procedure to be employed. Differentiation of intra- and extra hepatic cholelithiasis is the primary task for adequate management, so the site and nature of the obstructing lesion must be determined by means of imaging ${ }^{[5]}$.

Ultrasonography is the least invasive and least expensive imaging method. Multidetector computed tomography (MDCT) with good reformatting techniques has excellent accuracy in the evaluation of obstructive jaundice with regards to the level and cause of obstruction, but it requires injection of intravenous contrast medium \& the patient is exposed to ionizing radiation risks $^{[6]}$.

The development of fast imaging sequences and the improvement in the quality of abdominal images have generated a new interest in magnetic resonance evaluation of biliopancreatic diseases ${ }^{[7]}$.

Magnetic

resonance cholangiopancreatography (MRCP) is a 
completely non-invasive technique that provides good projectional images without administration of contrast agents, without radiation, requires no anaethesia and less operator- dependent. By using heavily T2weighted sequences, the signal of static or slow-moving fluid-filled structures such as the bile and pancreatic ducts is greatly increased, resulting in increased duct-tobackground contrast. So signals from the fluid in the biliary system and pancreatic duct are hyperintense, whereas signals from the background tissue are hypointense, enabling excellent depiction of the biliary and pancreatic systems. MRCP protocols applied for imaging of the hepatobiliary system were as follows: T2-weighted fast spin echo sequence on the axial and coronal planes; three-dimensional, fat suppressed, heavily T2-weighted fast spin echo sequence with multislab acquisition mode; twodimensional thick single slab projectional images; and three-dimensional reconstruction algorithms. ${ }^{[8] .}$

It has been demonstrated that the clinical value of using MRCP is similar to that of diagnostic direct cholangiopancreatography and in most instances, MRCP will gradually replace direct cholangiopancreatography for diagnostic purposes and provides an efficient alternation when diagnostic ERCP or PTC is unsuccessful or inadequate ${ }^{[9] .}$

MRCP usually enables the identification of normal and abnormal post-operative changes. In cases of complete obstruction of the bile duct, it allows analysis of the biliary tract above and below the level of the obstruction, a capability essential for treatment planning and one that is not provided by either ERCP or PTC ${ }^{[10]}$.

\section{Patients and Methods:}

This study included 20 patients; 11 males and 9 females. Their age ranged between 35 to 83 years. The prospective study protocol was approved by the ethical committee.

The included patients were referred from Shebin-Elkom fever hospital and National liver institute to do MRCP for better evaluation, during the period from April 2019 to October 2020 with Malignant biliary obstruction, prothrombin concentration > $>0 \% \%$, Platelet count > 50,000 platelet $/ \mathrm{ml}$.

Patients with contraindication to MRI (e.g.: patients who have heart pacemaker, metallic foreign body and metallic device with severe claustrophobia) or patients with benign biliary obstruction, serious coagulation 
disorder, renal impairment, ascites were excluded.

All the patients were subjected to full history taking, clinical examination, laboratory investigations (CBC, liver function tests, Prothrombin time, renal function tests, serum electrolytes and quantitative tumor markers).

\section{Imaging modalities:}

1- Abdominal ultrasound examination (Toshiba-Nemio XG, GE LOGIQ P6 and Toshiba-Xario, 2000).

2- MRCP.

3- A contrast enhanced Multi-detector computed tomography

(MDCT) examination of the abdomen and pelvis or triphasic CT of the abdomen and pelvis using a helical CT scanner (Siemens biograph dual source 64 slice).

The final diagnosis was reached through correlation with biopsy, tumor markers \& follow up imaging.

Imaging analysis: In all examined cases MRCP images were evaluated for:

* Possible cause of biliary obstruction.

* Level of biliary obstruction.

* Degree of biliary obstruction.
* Degree of dilatation of the intra and extra hepatic bile ducts.

The parameters used for MRCP. (Table 1)

\section{Statistical analysis:}

The results were collected, tabulated, and statistically analyzed with an IBM compatible personal computer with SPSS statistical package version 25 (SPSS Inc., Chicago, Illinois, USA). Descriptive statistics were used for example, number and percent for qualitative data, and mean and SD for quantitative data.

\section{Results:}

Our study included 20 patients 11 males and 9 females ranging from $35 y$ years to 83 years . Elevated bilirubin level both total and direct bilirubin were seen at all the cases. Total bilirubin levels were ranging from 3.6 to 43.5. Direct bilirubin levels were ranging from 1.9 to 30.6 . (figure 1)

Cholangiocarcinoma included the largest number of patients 12 out of 20 cases (60\%), while 4 cases out of 20 cases (20\%) presented with the pancreatic head carcinoma, one case out of 20 cases (5\%) presented with periamullary carcinoma, one case out of 20 cases $(5 \%)$ presented with hepatocellular carcinoma causing biliary 
obstruction, one case out of 20 cases presented with gall bladder carcinoma (5\%) and one case out of 20 cases (5\%) presented with gastric carcinoma. (figure 2).

Our study revealed that hailer biliary obstruction is more common than distal type about 14 cases out of 20 cases (70\%). (figure 3)

MRCP detected one case from 20 cases with incomplete biliary obstruction (5\%) and 19 cases with complete biliary obstruction. (figure4)

MRCP detected other related malignant features in 8 of 20 cases (40\%). Four cases out of 20 cases (20\%) with regional lymph nodal deposits, Two cases out of 20 cases $(10 \%)$ with portal vein invasion, one case out of 20 cases (5\%) with hepatic deposits and one case out of 20 cases (10\%) with peritoneal deposits. (figure 5)

The CBD diameter in our study is ranging from $3.9 \mathrm{~mm}$ to $32 \mathrm{~mm}$. The $\mathrm{CBD}$ is dilated at 5 cases of 20 cases $(25 \%)$ and it is of normal diameter at 15 cases of 20 cases (75\%). (figure 6)

Case 1: 53 years old female patient presented by abdominal pain, vomiting and gradual progressive jaundice.

\section{CT findings (Figure 7A):}

(A) Axial CT with contrast: Polypoidal enhancing mural thickening at the gastric pyloric region measuring $2 \mathrm{~cm}$ (Red arrow).

\section{MRCP findings (Figure $7 \mathrm{~B} \& \mathrm{C}$ ):}

(B) Coronal $3 D$ with MIP: There is moderate dilatation of the intrahepatic biliary radicles, right and left hepatic ducts and proximal common hepatic ducts (Bismuth I) (Red arrow).

(C) Axial T2WI: There is moderate dilatation of the intrahepatic biliary radicals (Red arrow). 


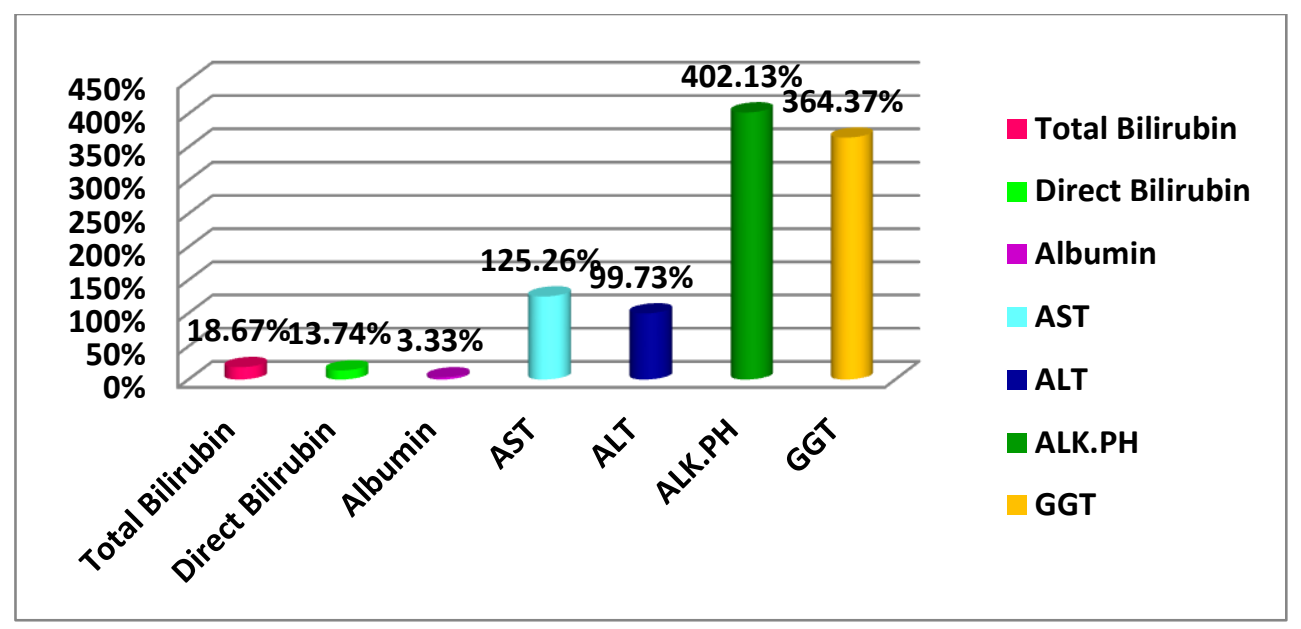

Figure (1) Serum bilirubin level and liver enzymes among the studied cases

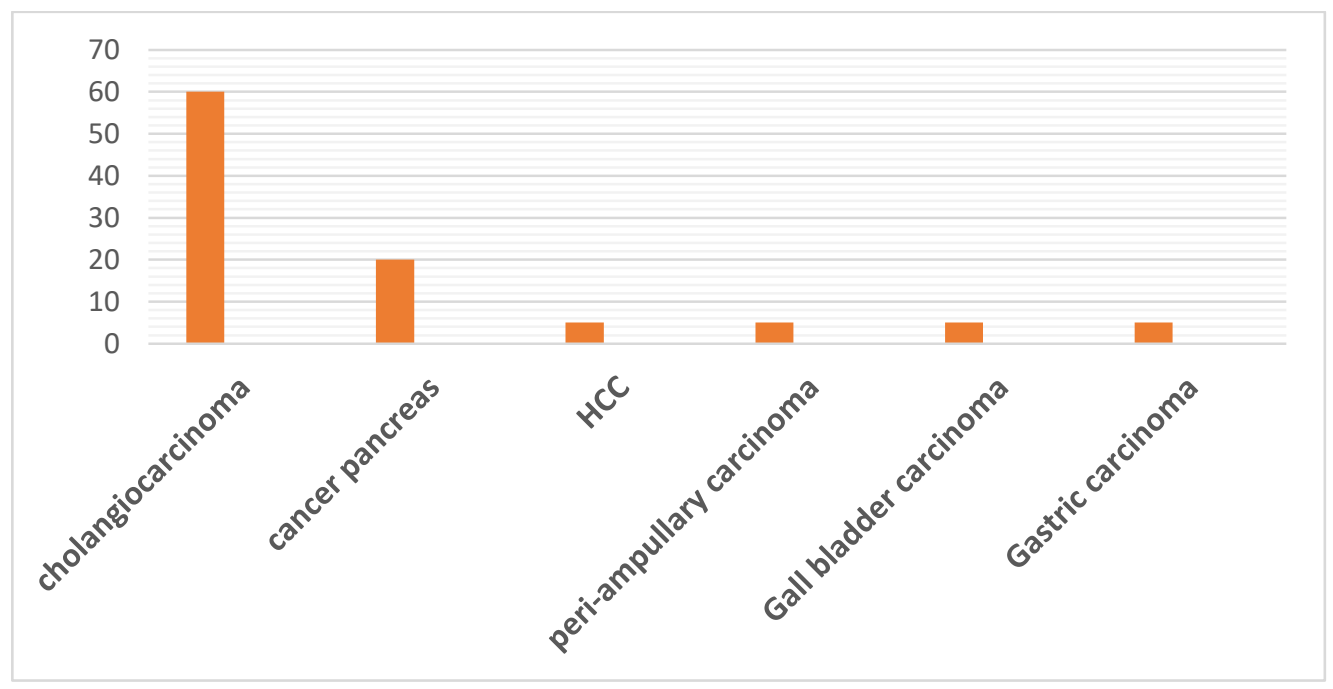

Figure (2): Causes of obstruction among the studied cases

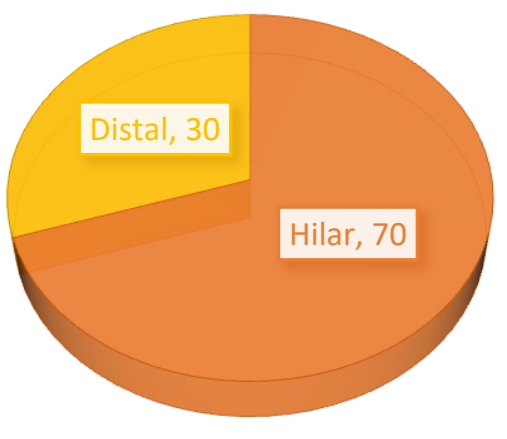

Figure $\left({ }^{(}\right)$: Level of biliary obstruction among the studied groups 


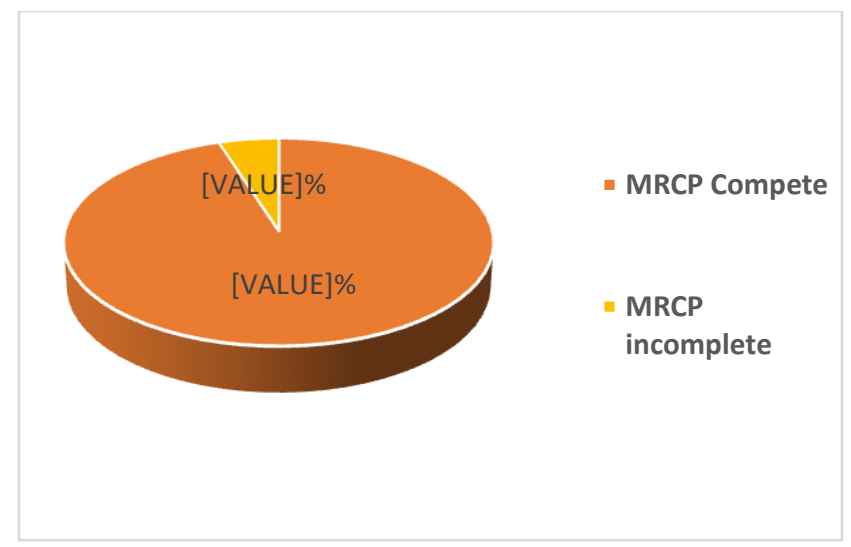

Figure (4): The detectability of the degree of obstruction among the studied groups by MRCP.

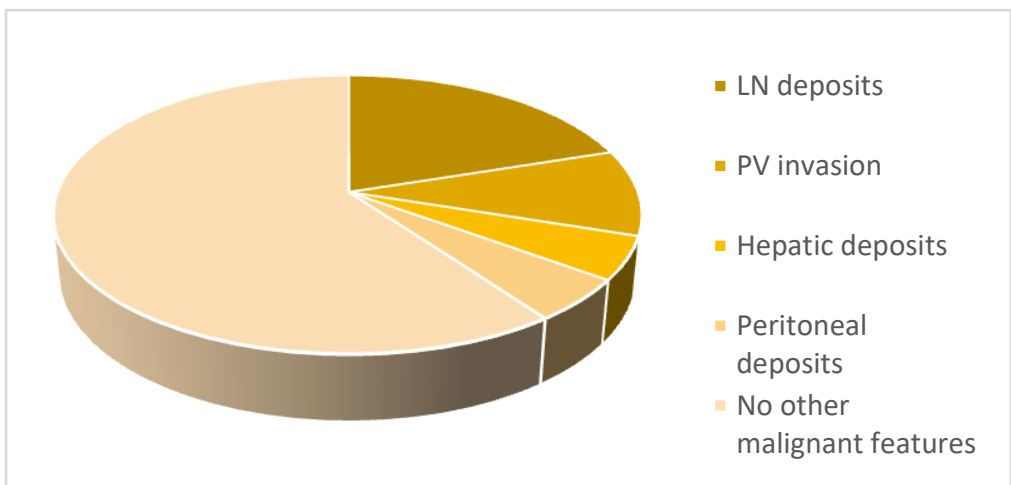

Figure (5): The other related malignant features detected by MRCP.

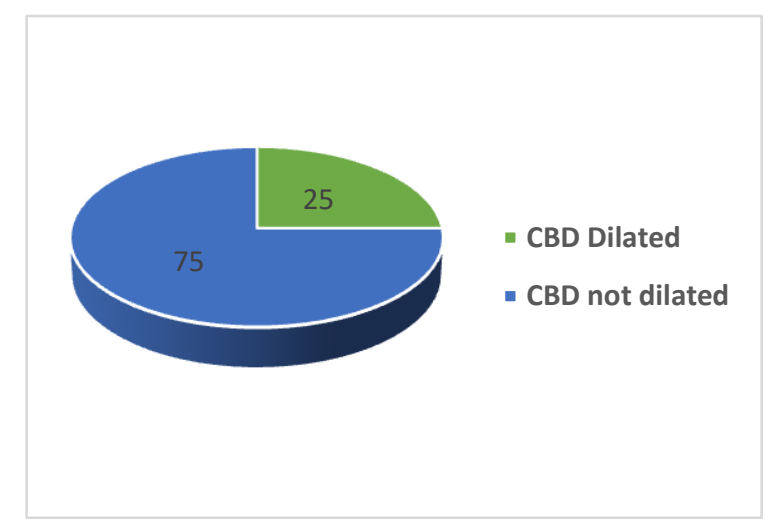

Figure (6): The CBD diameter among the studied groups. 

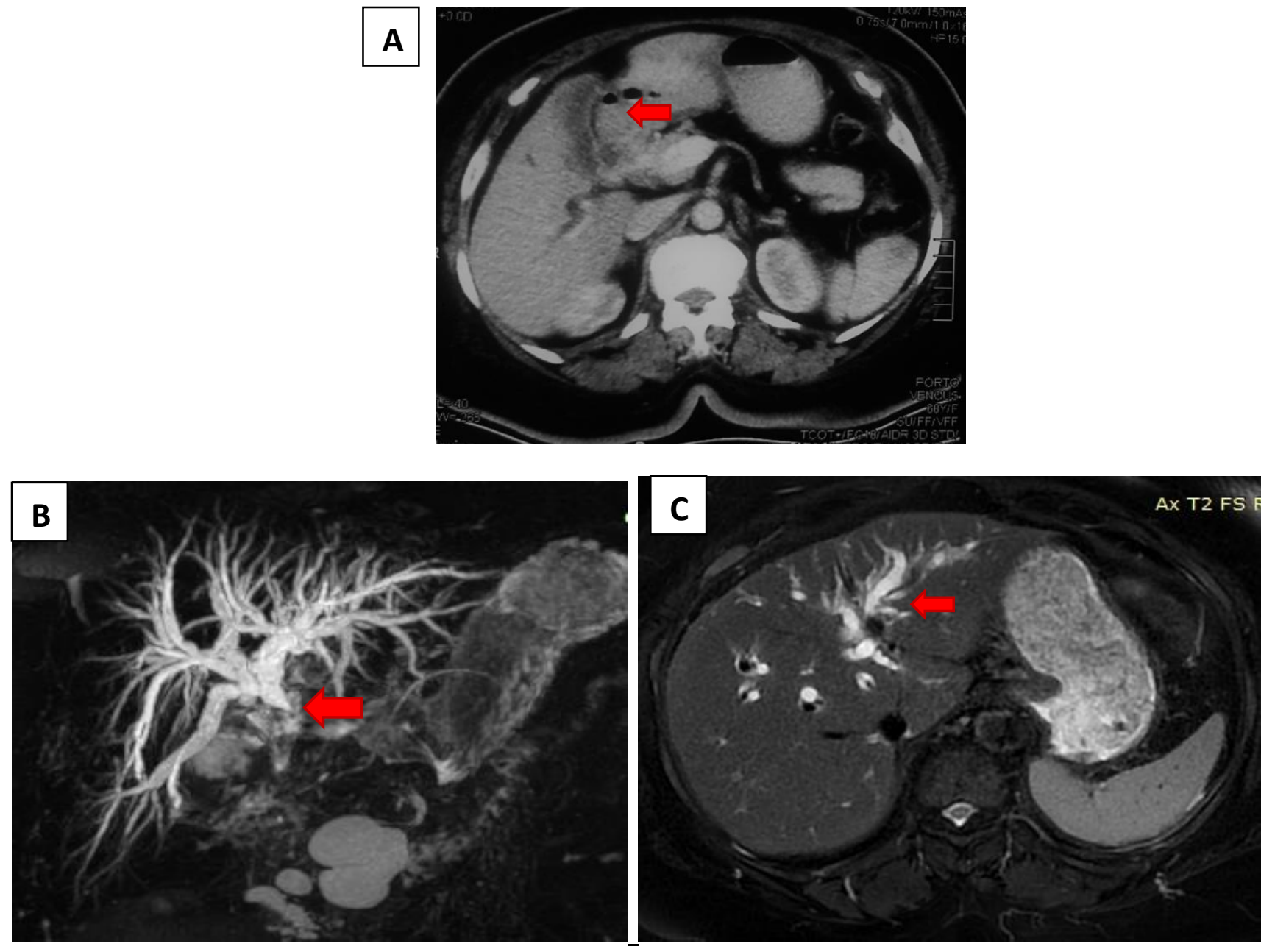

Figure7:(A) Axial CT with contrast: Polypoidal enhancing mural thickening at the gastric pyloric region measuring $2 \mathrm{~cm}$ (Red arrow). (B) Coronal 3D with MIP: There is moderate dilatation of the intrahepatic biliary radicles, right and left hepatic ducts and proximal common hepatic ducts (Bismuth I) (Red arrow). (C) Axial T2WI: There is moderate dilatation of the intrahepatic biliary radicals (Red arrow).

Table 1 : parameters of MRCP

\begin{tabular}{lllllll}
\hline & $\begin{array}{l}\text { Axial heavy } \\
\text { T2 }\end{array}$ & $\begin{array}{l}\text { Axial T2 } \\
\text { propeller fat } \\
\text { sat }\end{array}$ & $\begin{array}{l}\text { Coronal } \\
\text { 3D thin } \\
\text { cuts }\end{array}$ & $\begin{array}{l}\text { Axial 3D thin } \\
\text { cuts }\end{array}$ & Coronal 2D & $\begin{array}{l}\text { Axial } \\
\text { diffusion }\end{array}$ \\
\hline $\begin{array}{l}\text { Patient } \\
\text { position: }\end{array}$ & Supine & Supine & Supine & Supine & Supine & Supine \\
FOV : & 38 & 38 & 36 & 32 & 36 & 38 \\
Slice number: & 30 & 30 & 100 & 84 & 30 & 25 \\
Slice & $6 \mathrm{~mm}$ & $6 \mathrm{~mm}$ & $1 \mathrm{~mm}$ & $2 \mathrm{~mm}$ & $5 \mathrm{~mm}$ & $5 \mathrm{~mm}$ \\
thickness: & & & & & & \\
TR: & $14000 \mathrm{msec}$ & $2000 \mathrm{msec}$ & $2000 \mathrm{msec}$ & $3000 \mathrm{msec}$ & $2727 \mathrm{msec}$ & $8700 \mathrm{msec}$ \\
TE: & $300 \mathrm{msec}$ & $95 \mathrm{msec}$ & $529 \mathrm{msec}$ & $463 \mathrm{msec}$ & $102 \mathrm{msec}$ & $57 \mathrm{msec}$ \\
Scan time: & $1: 30 \mathrm{~min}$ & $5 \mathrm{~min}$ & $4 \mathrm{~min}$ & $2: 35 \mathrm{~min}$ & $2: 44 \mathrm{~min}$ & $1: 27 \mathrm{~min}$ \\
Matrix size: & $256 \times 192$ & $320 \times 320$ & $288 \times 288$ & $224 \times 224$ & $320 \times 320$ & $92 \times 128$ \\
NEX: & 1 & 1.5 & 1 & 1 & 1 & 1 \\
\hline
\end{tabular}




\section{Discussion:}

In our study, 20 patients were subjected to MRCP examination. MRCP examinations were performed using breath- hold 2D single-slice FSE and non-breath- hold multislice acquisition followed by 3D MIP reconstruction with complementary axial or coronal images.

The $r \cdot$ examined cases were classified into 7 groups according to malignant cause of biliary obstruction.

Cholangiocarcinoma included the largest number of patients 12 cases $(60 \%)$, while four cases $(20 \%)$ presented with pancreatic head carcinoma, one case $(5 \%)$ presented with periamullary carcinoma, one case $(5 \%)$ presented with hepatocellular carcinoma causing biliary obstruction, one case presented with gall bladder carcinoma (5 $\%$ ) and one case (5\%) presented with gastric carcinoma. It was concluded in study done in $2017^{[11]}$ that cholangiocarcinoma was the commonest cause found, 22 out of 43 cases $(51.2 \%), 4$ cases presented with pancreatic head carcinoma (9.3\%), 4 cases presented with hepatocellular carcinoma (9.3\%), 4 cases presented with distal stricture $(9.30 \%), 5$ cases presented with periampullary carcinoma (11.6\%), 1 case $(2.32 \%)$ presented with each of lymphoma,
GB carcinoma, liver metastasis from gastric neoplasm and suprarenal carcinoma. In our study, MRCP was accurate in the depiction the level of obstruction in the all 20 cases. It was concluded in the study done in $2014^{\text {[5] }}$ that MRCP showed 100\% sensitivity, specificity and diagnostic accuracy in detecting periampullary carcinoma. The study performed in $2017^{\text {[11] }}$ showed that MRCP was accurate in detecting the level of obstruction in all the studied 43 cases.

Obstruction was considered hailer if the lesion was at the confluence of the right and left hepatic ducts until $2 \mathrm{~cm}$ distal to the confluence. Obstruction was considered distal if the lesion was distal more than $2 \mathrm{~cm}$ from the confluence. Obstruction was considered of variable levels when there was more than one level of biliary obstruction detected at the time of MRCP examination.

Through our study, there were different levels of biliary obstruction detected by MRCP among the 20 cases presented with biliary obstruction. Obstruction was hailer in 14 cases $(70 \%)$ and obstruction was distal in 6 cases $(30 \%)$.

While in a study done in $2003^{[12]}$, among the 82 patients with pancreato-biliary 
diseases, $9.7 \%, 73 \%$ and $17 \%$ of cases had pancreato-biliary obstructive locations in intra-hepatic, extra-hepatic bile duct and main pancreatic duct, respectively.

In our study, cases with hailer obstruction are classified according to Bismuth classification into I, II, IIIa, IIIb and IV, while in the study done in $2014^{[13]}$, cholangiocarcinoma (CCA) cases are classified based on their anatomic location as intrahepatic CCA (iCCA), perihailar CCA (pCCA) and distal (dCCA) subtypes.

In our study the incidence of Bismuth type IV among the studied cases was the most common Bismuth type, 8 cases of the studied 20 cases $(40 \%)$. While the study done in $2014^{[13]}$ stated that extra-hepatic cholangiocarcinoma (90\%) is more common than intra-hepatic cholangiocacinoma (10\%).

Our study showed that MRCP with $100 \%$ accuracy in detecting the level of obstruction and MRCP showed significant preference in the ability to detect the cause of malignant obstruction, MRCP could diagnose the cause of malignant obstruction in 18 cases (90\%). Our study agreed with the study done in $2009,{ }^{[14]}$ which showed that MRCP could detect the level in all cases of obstruction at the porta and supra-pancreatic levels and at the intra-pancreatic level, MRCP was able to correctly diagnose all cases of cholangiocarcinoma and periampullary carcinoma but it could not detect one case each of carcinoma head of pancreas and carcinoma gall bladder.

The study performed in $2009^{[14]}$ showed that MRCP was able to detect the level of obstruction in $95.45 \%$ and the cause in $87.50 \%$ cases, agreement with our study. MRCP detected one case from 20 cases with incomplete biliary obstruction (5\%).

The study performed in $2000^{[15]}$ showed that the sensitivity, specificity, and accuracy of MRCP can be increased 17\%-20\% when $\mathrm{T} 1$ and T2 WI are combined with MRCP images for differentiation of benign from malignant causes of biliary dilatation. In our study conventional MR images played an important role, helping in the diagnosis of malignant biliary obstruction as regards detecting the site of malignant biliary lesion with its intra- or extra hepatic biliary ductal extension and the presence of associated metastasis, lymphadenopathy, organomegally, fluid collections and its location.

The study done in $2015^{[17]}$ concluded the sensitivity of $98 \%$ and specificity of $100 \%$ in detecting the malignant strictures by 
MRCP. Within our study, MRCP detected other related malignant features in $40 \%$ of cases, $20 \%$ with regional lymph nodal deposits, $10 \%$ with portal vein invasion, $5 \%$ with hepatic deposits and 5\% with peritoneal deposits while the study done in $2015^{\text {[17] }}$ study detected other malignant features in $50 \%$ of cases (lymph nodal deposits).

In spite of the several advantages of MRCP, there were also some limitations encountered in our study including: Relatively high cost, the possibility of claustrophobia, its inability to offer therapeutic intervention as compared with PTC or ERCP, the obtained image quality can be degraded by many factors including, marked obesity, massive ascites and inability to maintain breath holding in breath hold technique.

The examined cases in our study were referred either; when ERCP was contraindicated or not applicable to the patient or when there was technical failure of ERCP.

Finally, from our study we found that MRCP has several advantages as a developing technique in the evaluation of patients with malignant biliary obstruction as follow: MRCP is a non-invasive technique that provides projectional images similar to that of PTC and ERCP without administration of contrast agents also use no radiation, requires no medications, less operator dependent and no complications were reported in adequately screened patients, MRCP allows multiplanner views of the bilio-pancreatic tract without the need to mobilize the patients, MRCP provides global presentation of the pancreatico-biliary ductal system, both proximal and distal to the site of the biliary obstruction, MRCP can detect the nature of the obstructing lesion at the site of the obstruction, MRCP can be used in the cases of technical limitations of PTC and ERCP and as a part of complete MR examination, extends the diagnostic information from the bilio-pancreatic tract morphology to the surrounding structures.

\section{Conclusion:}

MRCP is superior as a non-invasive diagnostic tool in the diagnosis of malignant cause of biliary obstruction, the level of biliary obstruction and the other related malignant features with no substantial complications. MRCP is integrative modality in the diagnosis of malignant obstructive jaundice and providing the basis for the suitable further therapeutic procedures. 


\section{References:}

1. Matthew V., Scott P. \& Aaron S.: Evaluation of Jaundice in Adults. Am Fam Physician. 2017 Feb 1;95(3):164-168.

2. Motohara T., Semelka R. and Bader T: MR cholangio-pancreaticography. RadiolClin North Am 2003;41(1):89-96 .

3. Rösch T, Meining A, Frühmorgen S, Zillinger C, Schusdziarra V, Hellerhoff K. et al. A prospective comparison of the diagnostic accuracy of ERCP, MRCP, CT, and EUS in biliary strictures. Gastrointest Endosc. 2002 Jun;55(7):870-6. doi:10.1067/mge.2002.124206. PMID: 12024143.

4. Katabathina VS, Dasyam AK, Dasyam N, Hosseinzadeh K. Adult bile duct strictures: role of MR imaging and MR cholangiopancreatography in characterization. Radiographics. 2014 MayJun;34(3):565-86. doi: 10.1148/rg.343125211. PMID: 24819781.

5. Singh A, Mann HS, Thukral CL, Singh NR. Diagnostic Accuracy of MRCP as Compared to Ultrasound/CT in Patients with Obstructive Jaundice. J Clin Diagn Res. 2014 Mar;8(3):103-7. doi: 10.7860/JCDR/2014/8149.4120. Epub 2014 Mar 15. PMID: 24783094; PMCID: PMC4003596.

6. Mathew RP, Moorkath A, Basti RS, Suresh HB. Value and Accuracy of Multidetector Computed Tomography in Obstructive Jaundice. Pol $J$ Radiol. 2016;81:303-309. Published 2016 Jun 28. doi:10.12659/PJR.896680.
7. Vitellas KM, Keogan MT, Spritzer CE, Nelson RC. MR cholangiopancreatography of bile and pancreatic duct abnormalities with emphasis on the single-shot fast spin-echo technique. Radiographics. 2000 Jul-Aug;20(4):939-57; quiz 1107-8,

1112. doi:10.1148/radiographics.20.4.g00j123939.

Erratum in: Radiographics 2000 SepOct;20(5):1494. PMID: 10903685.

8. Liang C, Mao H, Wang Q, Han D, Li Yuxia L, Yue J. et al. Diagnostic performance of magnetic resonance cholangiopancreatography in malignant obstructive jaundice. Cell Biochem Biophys. 2011 Nov;61(2):383-8. doi: 10.1007/s12013-011-91953. PMID: 21567133.

9. Reinhold C, Bret PM. (2006): Current status of MR cholangiopancreatography. Am accuracy of ERCP, MRCP, CT, and EUS in biliary strictures. Gastrointest Endosc;5:870-6.

10. Hoeffel C, Azizi L, Maite' L, Laurent V, Aube' C, Arrive' L. et al., (2006): Normal and pathologic features of the postoperative biliary tract at 3-D MR cholangiopancreatography and MR imaging. RadioGraphics; 26:1603-20.

11. Ali ZA, Zytoon AA, Hady MA. Magnetic resonance cholangiopancreatography in malignant obstructive jaundice. Menoufia Medical Journal. 2017;30(1):110.

12.Zhong L, Yao QY, Li L, Xu JR. Imaging diagnosis of pancreato-biliary diseases: a control study. World J Gastroenterol. 2003 Dec;9(12):2824-7. doi: 10.3748/wjg.v9.i12.2824. PMID: 14669343; PMCID: PMC4612062. 
13.Hennedige TP, Neo WT, Venkatesh SK. Imaging of malignancies of the biliary tract- an update. Cancer Imaging. 2014 Apr 22;14(1):14. doi: 10.1186/1470-7330-14-14. PMID: 25608662; PMCID: PMC4331820.

14. Shukla V., Upadhyaya V., Upadhyaya D. Comparative assessment of imaging modalities in biliary obstruction. Indian Journal of Radiology and Imaging. 2009; 16(4), 577. malignant causes--value of adding conventional MR imaging to MR cholangiopancreatography. Radiology. $2000 \quad$ Jan;214(1):173-81. doi: 10.1148/radiology.214.1.r00ja35173. PMID: 10644119.

16. Madhok R., Rastogi S. Role of 3.0 Tesla MRCP in Obstructive Jaundice with Cyto/Histopathological or Surgical Correlation. 2015; 3(2):1-7.

15. Kim MJ, Mitchell DG, Ito K, Outwater EK. Biliary dilatation: differentiation of benign from

To cite this article: Medhat M. Refat, Ahmed E Shaalan, Asmaa Sabry. Role of Magnetic Resonance Cholangiopancreatography in Malignant Obstructive Jaundice. BMFJ 2021; 38(2): 511-523. DOI: 10.21608/bmfj.2021.63039.1385 